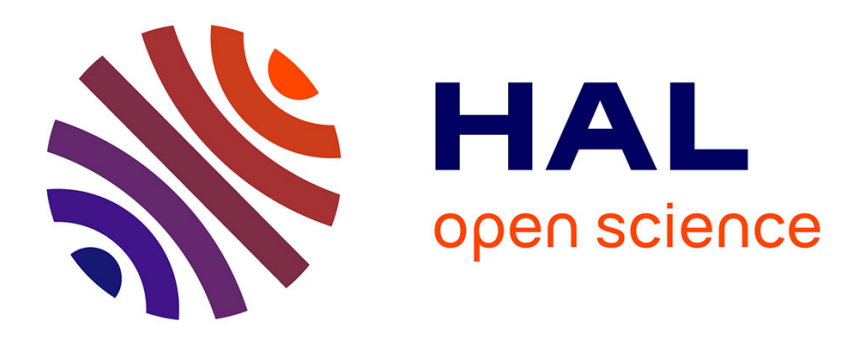

\title{
A hybrid identification and tracking of Lagrangian mesoscale eddies
}

\author{
Anass El Aouni
}

\section{To cite this version:}

Anass El Aouni. A hybrid identification and tracking of Lagrangian mesoscale eddies. Physics of Fluids, 2021, 33 (3), pp.036604. 10.1063/5.0038761 . hal-03161173

\section{HAL Id: hal-03161173 \\ https://hal.inria.fr/hal-03161173}

Submitted on 5 Mar 2021

HAL is a multi-disciplinary open access archive for the deposit and dissemination of scientific research documents, whether they are published or not. The documents may come from teaching and research institutions in France or abroad, or from public or private research centers.
L'archive ouverte pluridisciplinaire HAL, est destinée au dépôt et à la diffusion de documents scientifiques de niveau recherche, publiés ou non, émanant des établissements d'enseignement et de recherche français ou étrangers, des laboratoires publics ou privés. 


\section{A hybrid identification and tracking of Lagrangian mesoscale eddies}

Cite as: Phys. Fluids 33, 036604 (2021); https://doi.org/10.1063/5.0038761

Submitted: 27 November 2020 . Accepted: 31 January 2021 . Published Online: 05 March 2021

Anass El Aouni

COLLECTIONS

F This paper was selected as Featured

\section{Physics of Fluids GALLERY OF COVERS}




\title{
A hybrid identification and tracking of Lagrangian mesoscale eddies $\mathrm{B}$
}

\author{
Cite as: Phys. Fluids 33, 036604 (2021); doi: 10.1063/5.0038761 \\ Submitted: 27 November 2020 - Accepted: 31 January 2021 . \\ Published Online: 5 March 2021
}

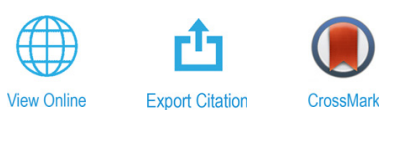

\author{
Anass El Aouni ${ }^{a)}$ (iD
}

\section{AFFILIATIONS}

Univ. Grenoble Alpes, Inria, CNRS, Grenoble INP, LJK, 38000 Grenoble, France

a) Author to whom correspondence should be addressed: anas.elaouni@gmail.com and anass.el-aouni@inria.fr

\begin{abstract}
Automatic identification and tracking of mesoscale eddies are crucial in large oceanic observational and numerical model data. This work proposes a fully automated method that identifies and tracks from a single Lagrangian advection, all mesoscale eddies, without prior knowledge of their lifespans. The eddies' detection and tracking use a hybrid method based on geometrical properties of the evolving velocity along Lagrangian trajectories and a grid density-based clustering algorithm. The high eddies' monitoring capacity of the proposed method is demonstrated by automatically identifying and tracking these structures from two different datasets: satellite-derived surface geostrophic velocity fields and a two-dimensional fluid simulation. The proposed approach gives complete dynamical features and evolution of the detected mesoscale eddies by identifying their genesis event, monitoring their coherent core, and describing their splitting and vanishing image.
\end{abstract}

Published under license by AIP Publishing. https://doi.org/10.1063/5.0038761

\section{INTRODUCTION}

The ocean is a fundamentally turbulent system that is characterized by the presence of several processes occurring at different scales, particularly mesoscale (ranging from $5 \mathrm{~km}$ up to several hundreds of kilometers), which is the most energetic scale in the ocean. The latter dominates the ocean's circulation by a variety of physical structures comprising eddies, meandering currents, filaments, and jets. The term mesoscale is often followed by the word "eddies," which reflects their predominance in the ocean. These physical structures form the main source of the ocean's kinetic energy ${ }^{1}$ and can have a crucial impact on it. In addition to their ability to stir and mix surrounding water, mesoscale eddies are also known for trapping and transporting momentum, heat, and different water properties. This can have an important impact on the regional and global distribution of water properties and on climate change, which arises from their effect on the circulation by transporting heat and salinity and also by exchanging momentum and extracting potential energy with/from the mean flow. ${ }^{2}$ For instance, mesoscale eddies off the Algerian basin are found to influence the regional mean circulation of the Levantine Intermediate Water. ${ }^{3}$ On a global scale, the authors in Ref. 4 show that global transport in the Southern Ocean is governed by the Agulhas eddies. The latter are found to carry and transport different water masses from the Indian ocean into the Southern Atlantic. In addition to their coherent transport properties, mesoscale eddies can deeply impact the primary production in the upper ocean, ${ }^{5-9}$ and their dynamics are found to partially control the displacement and foraging behavior of several marine inhabitants. ${ }^{10-12}$ Finally, their impact not only is limited to the near surface wind but also reaches cloud and rainfall. ${ }^{1}$

As the impact of mesoscale eddies on the oceanic biogeochemistry and regional and global circulation is remarkable, the development of fully automated methods with high eddy-monitoring capacity has recently received considerable interest in the field of oceanography. Several methods have been introduced in the literature to detect and monitor mesoscale eddies from observational and numerical velocity fields. One of the first works dedicated to eddies detection relies on the relative vorticity to identify rotating cores of vortices. ${ }^{14}$ This method was improved in Ref. 15 by introducing a wavelet technique to the relative vorticity. The authors in Ref. 16 attempt to explain some features of the movement of long-lived mesoscale eddies. The authors in Ref. 17 used learning approaches to investigate the closure of subgrid-scale stress for large-eddy simulations. A machine learning-based method is developed in Ref. 18 to detect vortices in complex flow. An $\omega_{R}$ criterion that computes the strength of the relative rotation has been developed in Refs. 19 and 20. The latter was modified in Ref. 21 to improve vortices identification and to cure the bulging phenomenon on the iso-surfaces caused by $\omega_{R}$. The authors in Ref. 22 consider a vortex as 
a homogeneous region characterized by a magnitude of vortex vector larger than zero. The authors in Ref. 23 introduced a universal gradient tensor decomposition of the vortex vector, which was used to detect rotational vortices. The work in Ref. 24 defines vortices as regions where eigenvalues of the velocity gradient tensor are complex. The authors in Ref. 25 proposed to use iso-surfaces of the eigenvalue's imaginary part to identify vortices. One of the widely used eddy detection methods in the oceanographic community is the Okubo-Weiss (OW) criterion, which separates flows into hyperbolic regions where strain dominates and elliptic ones where vorticity prevails. ${ }^{26,27}$ The authors in Ref. 28 introduced a vorticity curvature criterion, which is fundamentally based on the local properties of the vorticity field to detect vortices. These vorticity-based methods are very sensitive to an introductory threshold, which could lead to an under- or overquantification of eddies. $^{29,30}$ Moreover, these formulations are of Eulerian nature, which can strongly overestimate the degree of material coherency, most likely resulting in significant leakage occurring through their supposed detected eddies.

On the other hand, the Lagrangian perspective takes into account the time evolution of particles' trajectories, and therefore, it presents a robust basis to identify and track mesoscale eddies in a coherent manner. In the literature, several eddy definition and detection methods have been proposed within this perspective. For instance, based on the spinning movement of particles within vortices, the authors in Ref. 31 propose an approach that defines vortices from the frequency representation of their trajectories. The authors in Ref. 32 detect vortices based on a straightforward geometrical decomposition of their Lagrangian trajectories. The authors in Ref. 33 detected vortices as closed material barriers to the diffusive transport of vorticity. Another vorticity-based method is developed in Ref. 34, where they used the Lagrangian averaged vorticity deviation to detected coherent vortices. The authors in Ref. 35 trained a recurrent neural network on the time history of oceanic drifters for this purpose. The authors in Ref. 36 make use of the spectral clustering algorithm to identify vortices as Lagrangian trajectories that maintain short distances among themselves. The authors in Ref. 37 used a collection of eigenvalues and eigenvectors of spectral clustering and a Weyl-inspired eigengap to detect vortices. Other studies use probabilistic approaches that rely on the almost-invariant sets and probability density evolution. ${ }^{38,39}$ These methods provide satisfying results by sharply defining the eddies' boundaries and ensuring their coherency during their finite-time advection. The authors in Ref. 40 consider the relevant transfer operators and their infinitesimal generators on an augmented space-time manifold to detect coherent sets. However, they require the eddies' lifetime to be known in advance, which is usually the finite-time interval $\left[t_{0}, t_{f}\right]$ of the flow map $\Phi_{t_{0}}^{t}$. [The flow map $\Phi_{t_{0}}^{t}\left(\mathbf{x}_{0}\right):=\mathbf{x}\left(t, t_{0}, \mathbf{x}_{0}\right)$ is defined as the map that associates time $t_{0}$ and $t$ positions of a particle $\mathbf{x}_{0}$ that evolves according to a given velocity field $\left.\frac{d \mathbf{x}}{d t}=\mathbf{v}(\mathbf{x}, t)\right]$. That is, they only detect coherent cores of eddies with lifetimes at least equal to the finite-time duration of the flow map, by missing several other eddies that might occur during the finite-time $\left[t_{0}, t_{f}\right]$ interval but with shorter lifetimes. Moreover, these methods are based on the mapping of several Lagrangian quantities of the flow map $\Phi_{t_{0}}^{t_{f}}$ into a scalar field. Thus, they only detect the eddies that exist as coherent cores at the initial time $t_{0}$ of the flow map $\Phi_{t_{0}}^{t_{f}}$, therefore leaving undetected several other eddies that have merged right after the initial time $t_{0}$ somewhere within $] t_{0}, t_{f}[$.
In this work, we propose a new Lagrangian method that identifies and detects from a single run (advection of fluid parcels) all the coherent eddies regardless of their genesis time and lifetimes. The proposed method is based on the analysis of Lagrangian geometrical properties of the velocity fields and the use of a grid density-based clustering algorithm to identify and track their coherent cores. The proposed Lagrangian method is distinguished by the following features:

- it uses the notion of neighboring and therefore ensures the material coherency of its detected eddies.

- it does not require the eddies' lifetime to be equal to the time interval of advection.

- it does evaluate the state of the fluid at different sub-intervals from a single run. Therefore, it identifies and tracks Lagrangian eddies characterized by different lifespans.

- it does identify in a Lagrangian frame the genesis and splitting events of Lagrangian eddies, which usually impact the tracing of their trajectories and their estimated lifetimes.

The rest of this paper is structured as follows: Sec. II presents and details the proposed eddy identification and tracking method. Section III illustrates our method by identifying and tracking mesoscale eddies from two different velocity fields. Section IV validates the performance of the proposed method by comparing it with other methods. The conclusion is drawn in Sec. V.

\section{EDDY DETECTION METHOD}

The proposed method relies on the analysis of the Lagrangian geometrical properties of velocity fields and the use of a grid densitybased clustering algorithm. The method can be summarized in the three following steps: (i) it starts by segmenting Lagrangian trajectories into rotational and non-rotational segments based on the geometrical properties of their evolving velocities. (ii) It maps the initial and final position of the extracted rotational segments. (iii) It uses a grid density-based clustering algorithm to group them into different classes characterized by different transport properties, which represent the coherent cores of the eddies.

\section{A. Trajectory segmentation}

The proposed method is based on the analysis of the geometrical properties of evolving velocity fields. Angular momentum of a particle exhibiting rotation oscillates around a centerline with a given frequency, which presents the eddy-turn-over time, and with an increasing/decreasing Lagrangian angle, which represents the orientation of the eddy. This oscillation between negative and positive velocity vectors around its centerline allows an efficient characterization of particle rotation and orientation. We define coherent eddy as a group of adjacent particles that complete similar rotation around the same centerline and over the same interval of time. Every eddy is characterized by a genesis and splitting event, and between these two events, evolving velocities of its fluid parcels share similar geometrical properties. Outside of these events, its particles undergo different properties as they tend to follow different paths. Figure 1 shows an example of an evolving velocity of a particle within an eddy. The segment in blue color presents its trajectory before the genesis of the eddy, in red, its evolution as a coherent core, and finally in green, its evolution after the splitting event. The proposed method starts by segmenting particles' trajectories based on the analysis of the geometrical properties of 

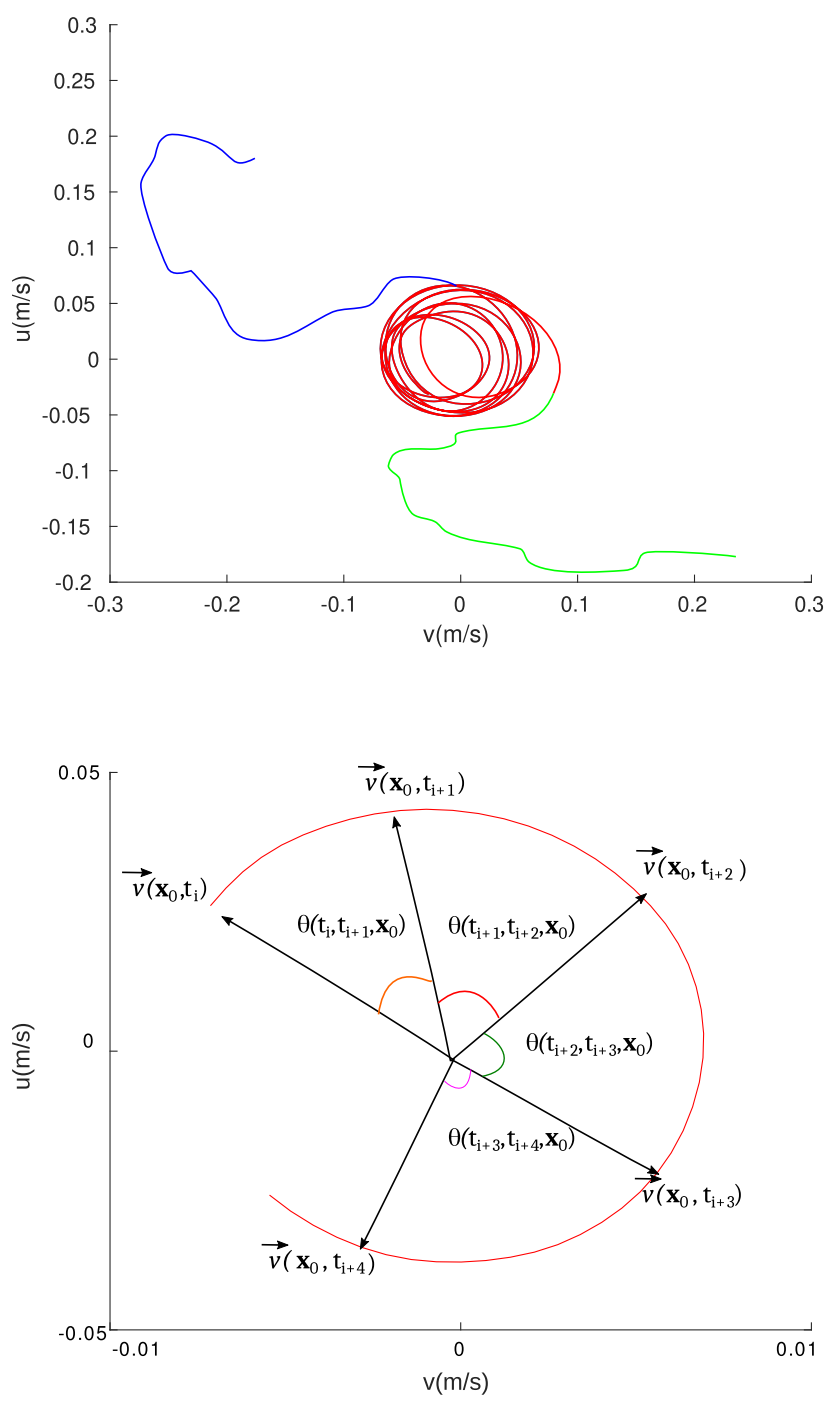

FIG. 1. (a) Evolving velocity along a Lagrangian trajectory of a particle within an eddy; the blue segment presents its evolution before the genesis of the eddy, red is between the genesis and splitting events, and the green segment represents its evolution after the splitting event. (b) A geometrical view of a rotational segment's identification.

their evolving velocities; each trajectory is divided into rotational segments [red segment in Fig. 1(a) as an example] and non-rotational ones [blue and green segments in Fig. 1(a) as an example].

We define a rotational segment $\mathbf{s}\left(t_{\beta}, t_{\alpha}, \mathbf{x}_{0}\right)$ of a trajectory $\mathbf{x}\left(t_{f}, t_{0}, \mathbf{x}_{0}\right)$ with $\left[t_{\alpha}, t_{\beta}\right] \in\left[t_{0}, t_{f}\right]$ as a segment characterized by an evolving velocity $\overrightarrow{\mathbf{v}}\left(t_{\beta}, t_{\alpha}, \mathbf{x}_{0}\right)$ that oscillates between negative and positive phases around a given centerline; the orientation of the angle between every two successive vectors,

$$
\theta\left(t_{i+1}, t_{i}, \mathbf{x}_{0}\right)=\cos ^{-1} \frac{\overrightarrow{\mathbf{v}}\left(\mathbf{x}_{0}, t_{i}\right) \cdot \overrightarrow{\mathbf{v}}\left(\mathbf{x}_{0}, t_{i+1}\right)}{\left|\overrightarrow{\mathbf{v}}\left(\mathbf{x}_{0}, t_{i}\right)\right|\left|\overrightarrow{\mathbf{v}}\left(\mathbf{x}_{0}, t_{i+1}\right)\right|}, \quad i \in[\alpha, \beta[,
$$

is the same along the segment. An example of a portion of such a segment is shown in Fig. 1(b). Every rotational segment $\mathbf{s}\left(t_{\beta}, t_{\alpha}, \mathbf{x}_{0}\right)$ is characterized by an initial time $t_{\alpha}$ and position $\mathbf{x}\left(t_{\alpha}, \mathbf{x}_{0}\right)$; final time $t_{\beta}$ and position $\mathbf{x}\left(t_{\beta}, \mathbf{x}_{0}\right)$; an orientation $\operatorname{sgn}\left(\theta\left(t_{\beta}, t_{\alpha}, \mathbf{x}_{0}\right)\right)$ such that $\forall \theta(t) \in \theta\left(t_{\beta}, t_{\alpha}, \mathbf{x}_{0}\right), \theta(t)>0$ or $\forall \theta(t) \in \theta\left(t_{\beta}, t_{\alpha}, \mathbf{x}_{0}\right), \theta(t)<0$; and a minimum time length $\left\|t_{\beta}-t_{\alpha}\right\|>m_{t}$. To filter out large rotational circulations, we only process rotational segments with a minimum angular momentum $\frac{\int_{t_{\alpha}}^{t_{\beta}} \theta\left(t, \mathbf{x}_{0}\right)}{\left(t_{\beta}-t_{\alpha}\right)}>\omega_{\pi}$.

\section{B. Rotational segment clustering}

Once rotational segments are extracted from all particles' trajectories, the next step consists of grouping them into different cores characterized by different transport properties. Rotational segments are clustered based on the following parameters:

- Rotational segments $\left\{\mathbf{s}_{i}\left(t_{i_{\beta}}, t_{i_{\alpha}}, \mathbf{x}_{i}\right)\right\}_{i=0}^{n}$ belonging to the same cluster share similar initial time $t_{\alpha}$ and adjacent positions $\left\{\mathbf{x}_{i}\left(t_{i_{\alpha}}, \mathbf{x}_{i}\right)\right\}_{i=0}^{n}$, which represent the initial position of the eddy right after its genesis event at $t_{\alpha} . \mathrm{n}$ is the number of rotational segments that create a given cluster.

- They also share similar final time $t_{\beta}$ and adjacent positions $\left\{\mathbf{x}_{i}\left(t_{i_{\beta}}, \mathbf{x}_{i}\right)\right\}_{i=0}^{n}$, which represent the final position of the eddy as the coherent core before its splitting event after $t_{\beta}$.

- Rotational segments within the same cluster share the same orientation representing the nature of the eddy (cyclone or anticyclone).

To achieve the aforementioned classification, we make use of a grid density-based clustering algorithm. ${ }^{41}$ We start by mapping these rotational segments into their initial and final time $t_{\alpha}, t_{\beta}$, respectively,

$$
\begin{gathered}
\left\{\mathbf{x}_{i}\left(t_{i_{\alpha}}\right), \mathbf{x}_{i}\left(t_{i_{\beta}}\right)\right\}_{i=0}^{n}=\left\{\Phi_{t_{0}}^{t_{t_{\alpha}}}\left(\mathbf{x}_{i}\left(t_{0}\right)\right), \Phi_{t_{0}}^{t_{i \beta}}\left(\mathbf{x}_{i}\left(t_{0}\right)\right)\right\}_{i=0}^{n}, \\
\left\{t_{i_{\alpha}}\right\}_{i=0}^{n} \in\left[t_{0}, t_{f}\left[, \quad\left\{t_{i_{\beta}}\right\}_{i=0}^{n} \in\right] t_{0}, t_{f}\right] .
\end{gathered}
$$

Then, we compute the density of each particle $p_{j}$ based on the number of its $\epsilon$-neighboring particles at its bordering time $t_{\alpha}$ and $t_{\beta}$ as

$$
\begin{aligned}
& N_{j \epsilon_{\alpha}}\left(\left\{\mathbf{x}_{i}\left(t_{i_{\alpha}}\right)\right\}_{i=1}^{n}, p_{j}\right)=\left\{p_{j}, p_{k} \in\left\{\mathbf{x}_{i}\left(t_{i_{\alpha}}\right)\right\}_{i=1}^{n}: d\left(p_{j}, p_{k}\right) \leq \epsilon\right\} \\
& N_{j \epsilon_{\beta}}\left(\left\{\mathbf{x}_{i}\left(t_{i_{\beta}}\right)\right\}_{i=1}^{n}, p_{j}\right)=\left\{p_{j}, p_{k} \in\left\{\mathbf{x}_{i}\left(t_{i_{\beta}}\right)\right\}_{i=1}^{n}: d\left(p_{j}, p_{k}\right) \leq \epsilon\right\},
\end{aligned}
$$

where $N_{j \epsilon}$ is a function that associates with each particle $p_{j}$ a given density based on its neighboring. $d\left(p_{j}, p_{k}\right)$ denotes the Euclidean distance between particles $p_{j}$ and $p_{k} . \epsilon$ is the minimum Euclidean distance for neighboring. We define a coherent eddy as a time-evolving domain,

$$
\mathbf{E}=\bigcup_{t \in\left[t_{\alpha}, t_{\beta}\right]} \mathbf{E}(t) \subset \mathbb{R}^{2} \times\left[t_{\alpha}, t_{\beta}\right],
$$

in a way that $\mathbf{E}$ is a set of adjacent particles $\left\{\mathbf{x}_{j}\right\}_{j=0}^{m}$ characterized by rotational segments that co-exist in the dense regions $G\left(t_{\alpha}\right)$ $=\left\{N_{j \epsilon_{\alpha}}\right\}_{j=0}^{m}$ and $G\left(t_{\beta}\right)=\left\{N_{j \epsilon_{\beta}}\right\}_{j=0}^{m}$, respectively, at $t_{\alpha}$ and $t_{\beta}$,

$$
\mathbf{E}=G\left(t_{\alpha}\right) \cap G\left(t_{\beta}\right) .
$$

The condition in Eq. (5) ensures the eddy's coherency, by including to the evolving core $\mathbf{E}(t)$ only particles that co-exist at both the genesis and splitting time of the eddy. By definition, a coherent eddy is a 
material domain, and its time evolution as a coherent core between $\left[t_{\alpha}, t_{\beta}\right]$ is expressed as

$$
\mathbf{E}\left(t_{\beta}, t_{\alpha}, \mathbf{E}_{\alpha}\right)=\left\{\left\{\mathbf{s}_{j}\left(t_{\beta}, t_{\alpha}, \mathbf{x}_{j}\right)\right\}_{j=0}^{m},\left\{\mathbf{x}_{j}\right\}_{j=0}^{m} \in \mathbf{E}\left(t_{\alpha}\right)\right\} .
$$

We express the evolution of its particles before the genesis event in terms of the flow map as

$$
\left.\left.\mathbf{E}(t)=\boldsymbol{\Phi}_{t_{\alpha}}^{t}\left(\mathbf{E}\left(t_{\alpha}\right)\right), t \in\right] t_{\alpha}, t_{0}\right]
$$

and the destination of its particles after splitting as

$$
\left.\left.\mathbf{E}(t)=\boldsymbol{\Phi}_{t_{\beta}}^{t}\left(\mathbf{E}\left(t_{\beta}\right)\right), t \in\right] t_{\beta}, t_{f}\right] .
$$

\section{RESULTS AND DISCUSSION}

To visually illustrate the proposed method, we chose to apply it on a fluid simulation characterized by the presence of 3 vortices and, therefore, provide a clear and full view of all its steps.

\section{A. Visual illustration of the method: Direct fluid simulation}

We start by numerically solving the Navier-Stokes equation for a two-dimensional velocity, $\nu: \mathcal{C} \rightarrow \mathbb{R}^{2}$, of an incompressible fluid on a periodic domain, $\mathcal{C}=[0,1] \times[0,1]$,

$$
\begin{aligned}
\partial \nu_{t}+\nu \cdot \nabla \nu & =-\nabla \rho+\frac{1}{R e} \Delta \nu+f, \quad(x, t) \in \mathcal{C} \times[\alpha, \beta], \\
\nabla \cdot \nu & =0, \quad(x, t) \in \mathcal{C} \times[\alpha, \beta], \\
\int \nu^{i} d x & =0, \quad(x, t) \in \mathcal{C} \times[\alpha, \beta], i=1,2, \\
\nu & =\nu^{*}, \quad(x, t) \in \mathcal{C} \times\{0\},
\end{aligned}
$$

with $\nu \cdot \nabla \nu$ being in control of kinetic energy transfer in the turbulent cascade. Incompressible flow is ensured by the pressure gradients $\nabla \rho$, and $\frac{1}{R e} \Delta \nu$ represents the viscous dissipation of fluid.

By presuming periodic boundary conditions, we numerically solve the Navier-Stokes model between the time interval $t$ $\in[-30,370]$ by using a standard pseudo-spectral method. We set the Reynolds number to $R e=10^{4}$ and use 128 modes in each direction with $2 / 3$ dealiasing. The time $t_{0}$ state of the system is characterized by the vorticity of a vortex $\left.\bar{\omega}\right|_{t_{0}}=-2 e^{\left(\frac{(x-\pi)^{2}+(y-\pi)^{2}}{0.4}\right)}$ perturbed by a random uniform distribution.

The model is parameterized by an external forcing $f=\cos \left(10^{-2} x+10^{-1} y\right)$ between $[-30,0[$ and then $f=0$ between $[0,370]$. We inject to the simulation the vorticity of two vortices $\left.\bar{\omega}\right|_{t_{30}}=-2 e^{\left(\frac{\left(x-\frac{2}{3} \pi\right)^{2}+\left(y-\frac{2}{3} \pi\right)^{2}}{0.1}\right)}$ and $\left.\bar{\omega}\right|_{t_{66}}=-2 e^{\left(\frac{\left(x-\frac{1}{3} \pi\right)^{2}+\left(y-\frac{1}{3} \pi\right)^{2}}{0.1}\right)}$, respectively, at $t=30$ and $t=66$. The vorticity stream formulation ${ }^{42}$ is used to extract the time-evolving velocity. Figure 2 displays the vorticity associated with the simulated velocity field of the Navier-Stokes model 9 captured at different time steps.

The method starts by producing particles' trajectories $\left\{\mathbf{x}_{j}\left(t_{f}, t_{0}, \mathbf{x}_{j}\right)\right\}_{j=0}^{m}$ that evolve according to the simulated velocity field of the Navier-Stokes model 9 between $t_{0}=0$ and $t_{f}=370$, which corresponds to the advection of $128 \times 128$ fluid parcels. Then, from these trajectories, it extracts rotational segments $\left\{\mathbf{s}_{i}\left(t_{i_{\beta}}, t_{i_{\alpha}}, \mathbf{x}_{i}\right)\right\}_{i=0}^{n}$ as
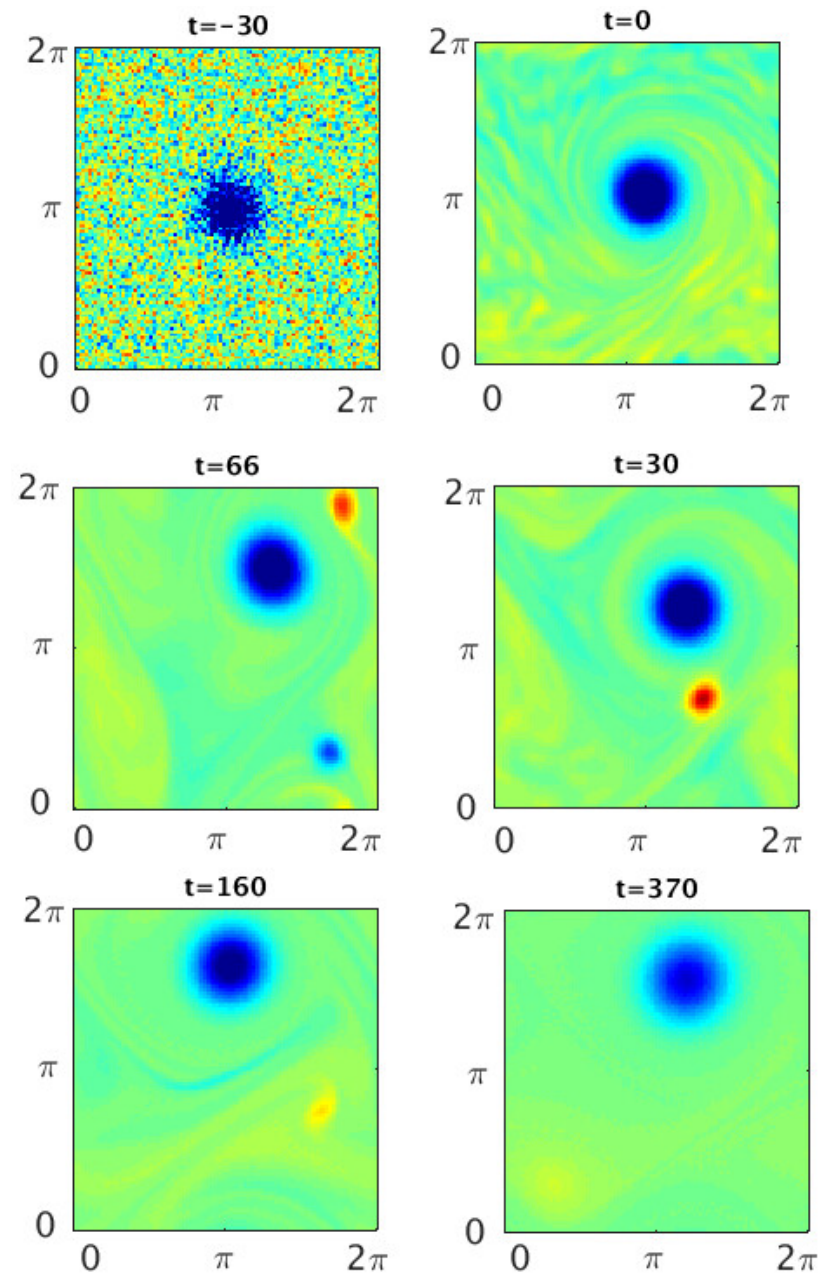

FIG. 2. Time evolution of the vorticity governed by the Navier-Stokes model 9 evaluated at times $t=(-30,0,30,66,160,370)$. Video M0 shows the time evolution of this fluid simulation. Multimedia view: http://dx.doi.org/10.1063/5.0038761.1

segments where their evolving velocities share the same orientation $\left\{\operatorname{sgn}\left(\theta\left(t_{i_{\beta}}, t_{i_{\alpha}}, \mathbf{x}_{i}\right)\right)\right\}_{i=0}^{n}$, following Eq. (1). We set the minimum time length $m_{t}$ to $30 \Delta t$ and $\omega_{\pi}=\frac{\pi}{400} \mathrm{rad} / \Delta t$ to reduce the number of processed segments and filter out large-scale rotations.

Once the rotational segments are extracted, the method proceeds by mapping the initial $\left\{\mathbf{x}_{i}\left(t_{i_{\alpha}}\right)\right\}_{i=1}^{n}$ and final $\left\{\mathbf{x}_{i}\left(t_{i_{\beta}}\right)\right\}_{i=1}^{n}$ positions of particles within these rotational segments as shown in Figs. 3(a) and 3(b). We set $\epsilon$ equal to three times of the grid resolution and compute for each distribution $\left\{\mathbf{x}_{i}\left(t_{i_{\alpha}}\right)\right\}_{i=0}^{n}$ and $\left\{\mathbf{x}_{i}\left(t_{i_{\beta}}\right)\right\}_{i=0}^{n}$ its corresponding density $\left\{N_{j \epsilon_{\alpha}}\right\}_{i=0}^{n}$ and $\left\{N_{j \epsilon_{\beta}}\right\}_{i=0}^{n}$ and identify regions $\left\{G\left(t_{i_{\alpha}}\right), G\left(t_{i_{\beta}}\right)\right\}_{i=0}^{i=k}$ with higher density distribution of particles as shown in Fig. 3(c). Each initial group $G\left(t_{i_{\alpha}}\right)$ representing the vortex's core right after its genesis is associated with a final group $G\left(t_{i_{\beta}}\right)$ representing the vortex's core right before its splitting event. To ensure the coherency of the vortices, we define their evolving 


$$
\left\{\mathbf{x}_{i}\left(t_{i_{\alpha}}\right)\right\}_{i=0}^{n}
$$

$$
\left\{\mathbf{x}_{i}\left(t_{i_{\beta}}\right)\right\}_{i=0}^{n}
$$
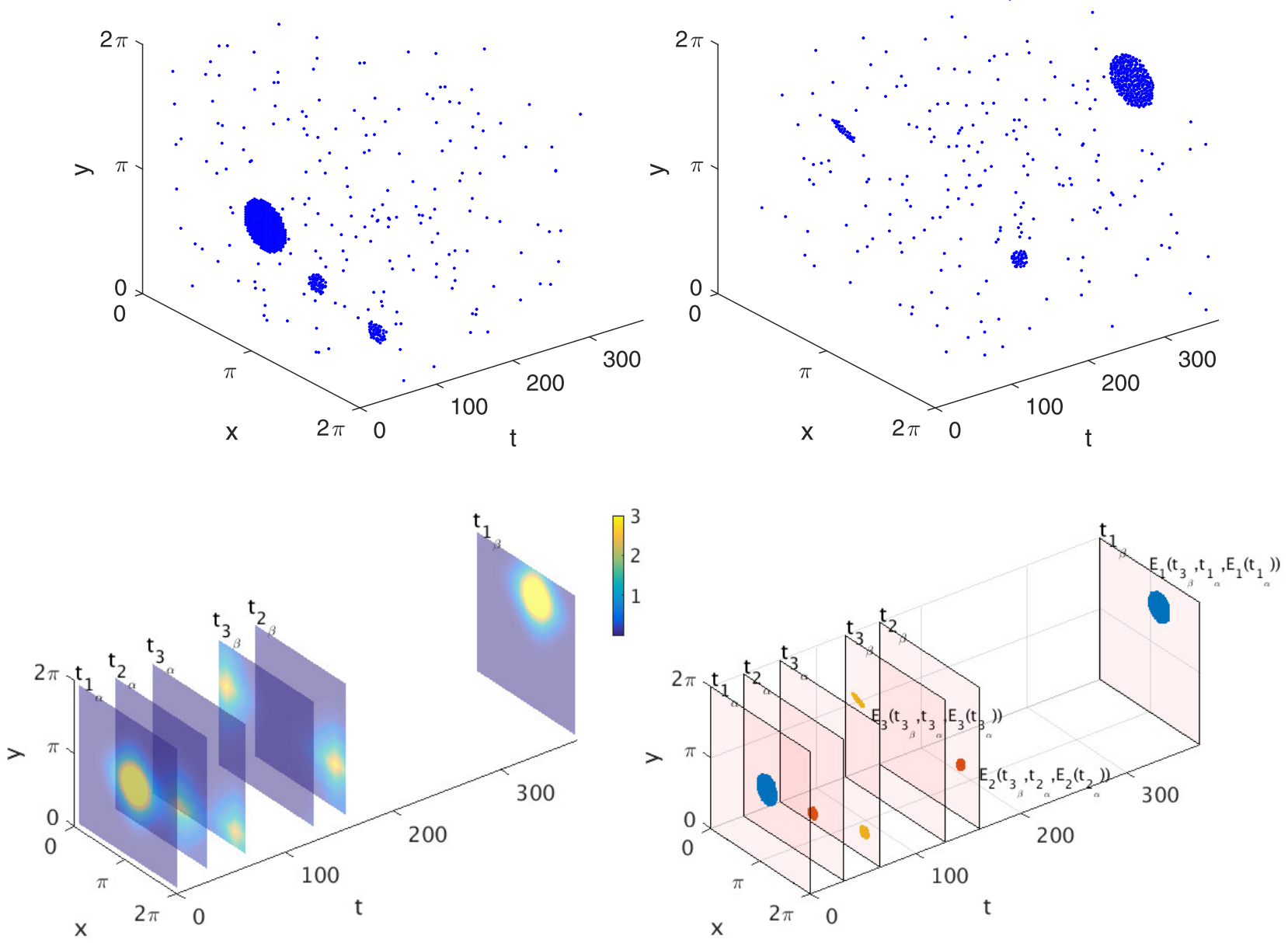

FIG. 3. (a) and (b) Initial/final positions of fluid particles within rotational segments at time $t_{\alpha} / t_{\beta}$, each cluster of neighboring points refers to a group of particles that share similar evolving rotations. (c) Density distribution of the initial and final positions of fluid particles within rotational segments. (d) Initial (at $t_{\alpha}$ right after the merging event) and final $\left(t_{\beta}\right.$ right before the splitting event) positions of the three extracted vortices presented in blue, red, and yellow colors.

cores as $\left\{\mathbf{E}_{\mathbf{i}}\right\}_{i=0}^{i=k}=\left\{G\left(t_{i_{\alpha}}\right) \cap G\left(t_{i_{\beta}}\right)\right\}_{i=0}^{i=k}$. Figure 3(d) shows the initial and final positions of the three detected vortices, presented in three different colors (blue, red, and yellow). These Lagrangian vortices are extracted from a single run, and they are characterized by different genesis times and lifetimes, showing the high vortex-monitoring capacity of the proposed method.

\section{B. Application to satellite geostrophic currents}

In this part, we make use of geostrophic currents derived from the satellite acquired sea surface height under the geostrophic approximation,

$$
\mathbf{v}(\phi, \theta, t)=g f^{-1} \nabla^{\perp} \eta(\phi, \lambda, t),
$$

where $g$ is the acceleration of gravity, $f$ presents the Coriolis coefficient, and $\eta(\phi, \lambda, t)$ is the sea surface height (SSH), which serves as a non- canonical Hamiltonian for surface velocity. $\perp$ stands for a $90^{\circ}$ anticlockwise rotation. These velocity data are provided daily with a spatial resolution of $1 / 4^{\circ}$ by the Copernicus Marine Environment Monitoring Service $(C M E M S)$. We collect these daily data for the period between January 1, 2006, and December 31, 2006, with a spatial domain covering the Northern Atlantic Ocean.

Here, we identify and track mesoscale eddies over a large domain $U$ to stretch the performance of the proposed method and its high eddy-monitoring capacity. We chose $U=\left[32^{\circ} \mathrm{N}, 17^{\circ} \mathrm{W}\right]$ $\times\left[22^{\circ} \mathrm{N}, 27^{\circ} \mathrm{W}\right]$ (blue box in Fig. 4), which lies off the Canary Current, a region characterized by high eddy kinetic energy ${ }^{43}$ and mesoscale eddies that frequently span off the islands. Within the North Atlantic ocean, we define a domain of advection $D$ (green box in Fig. 4) and set a regular grid $G_{D}$ of introductory conditions with a spatial resolution of $0.04^{\circ} \times 0.04^{\circ}$ over the year 2006. We set the advection time $t_{f}-t_{0}$ to 360 days with a time step of $8 \mathrm{~h}$ and use a bilinear interpolation method with a step size-adapting fourth-order 


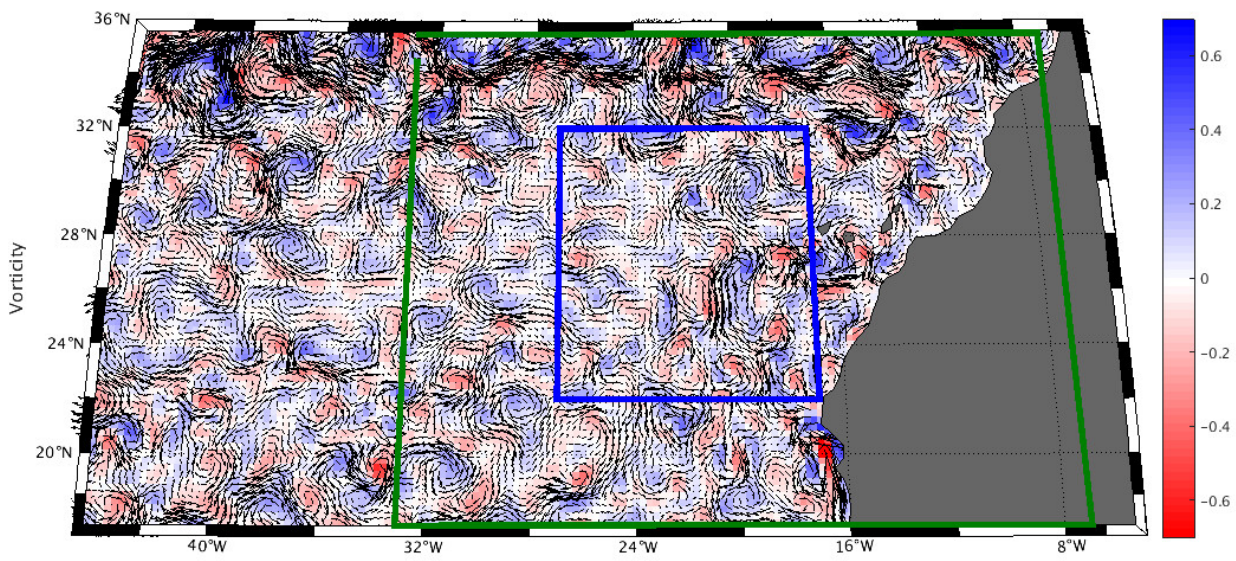

FIG. 4. Surface geostrophic velocity (vectors) and relative vorticity field over the Northern Atlantic Ocean. The blue box presents the domain used to identify and extract mesoscale eddies from the geostrophic current over the year 2006. The green box represents the domain of particles' advection.

Runge-Kutta to carry out all $250 \times 250$ particles' integration. We set the minimum time length $m_{t}$ to 15 days, $\omega_{\pi}=\frac{\pi}{4} \mathrm{rad} / \mathrm{month}$, and the neighboring minimum distance $\epsilon=0.12^{\circ}$.

The single run over 2006 results in detecting different eddies characterized by different initial times and lifetimes. To map all the detected eddies into one image, we chose to present them in different colors depending on their genesis time. Therefore, overlapping eddies with different colors are detected at different time $t \in\left[t_{0}, t_{f}[\right.$. A total of 64 mesoscale eddies were detected from a single run over 2006. They are characterized by lifetimes and diameters varying from 30 to 173 days and 21 to $110 \mathrm{~km}$, respectively. They reach a maximum speed of $7.7 \mathrm{~km}$ /day and can travel up to $728 \mathrm{~km}$ westward by self-advection due to the $\beta$-effect. ${ }^{4}$

We show in Fig. 5 the initial positions of all the 64 detected eddies $\left\{\mathbf{E}_{i}\left(t_{i_{\alpha}}\right)\right\}_{i=1}^{i=64}$ in closed curves, their trajectories, and their final positions $\left\{\mathbf{E}_{i}\left(t_{i_{\beta}}\right)\right\}_{i=1}^{i=64}$ (filled closed curves) before they lose their coherency. We show in video M1 (Multimedia view) the full evolution of these mesoscale eddies $\left\{\mathbf{E}_{i}\left(t_{i_{\beta}}, t_{i_{\alpha}}, \mathbf{E}_{i \alpha}\right)\right\}_{i=1}^{i=64}$ as coherent cores over their different lifetimes $\left\{\left[t_{i_{\alpha}}, t_{i_{\beta}}\right]\right\}_{i=1}^{i=64}$. These eddies maintain their coherency and do not stretch or fold, showing by that the high capacity of the method in sharply defines the eddies. We show in video M2 (Multimedia view) the evolution of the water forming these eddies and their genesis events $\left\{\mathbf{E}_{i}\left(t_{0}, t_{i_{\alpha}}, \mathbf{E}_{i \alpha}\right)\right\}_{i=1}^{i=64}$, and in a similar way, we show in video M3 (Multimedia view) the splitting events of the detected eddies $\left\{\mathbf{E}_{i}\left(t_{f}, t_{i_{\beta}}, \mathbf{E}_{i \beta}\right)\right\}_{i=1}^{i=64}$ and the destination of their trapped water, thereby displaying the ability of the method to precisely follow their dynamical evolution and long-term coherent water transport. Finally, we show in video M4 (Multimedia view) the full advection and interaction of all the detected eddies from genesis to

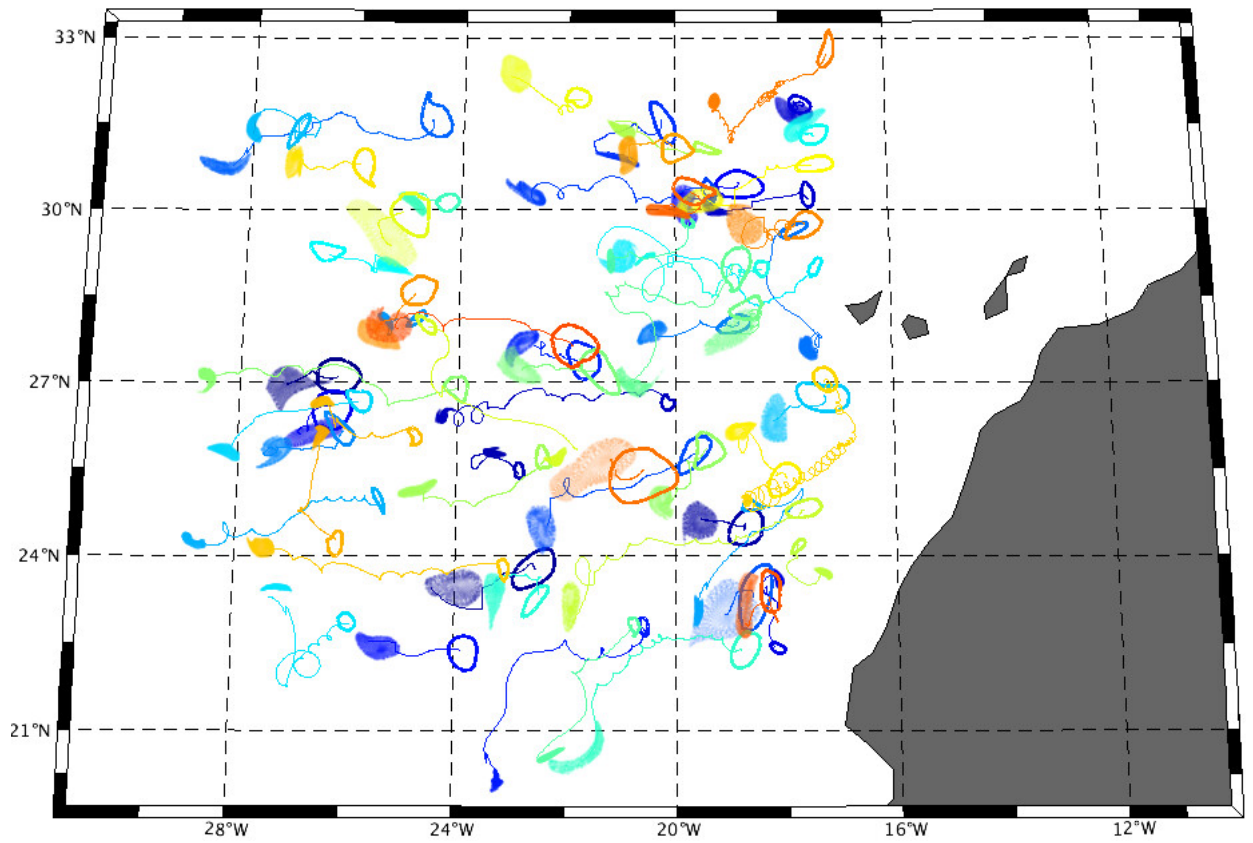

FIG. 5. Initial and final positions of all the 64 detected mesoscale eddies over the year of 2006. Mesoscale eddies are presented in different colors following their genesis time. Movie M1 shows the entire advection of the coherent cores of these mesoscale eddies, M2 shows their genesis events, and $\mathrm{M} 3$ shows their splitting events. Finally, video M4 shows the full interaction of their fluid parcels over 2006. Multimedia views: http://dx.doi.org 10.1063/5.0038761.2; http://dx.doi.org/ 10.1063/5.0038761.3; http://dx.doi.org/ 10.1063/5.0038761.4; http://dx.doi.org/ $10.1063 / 5.0038761 .5$ 
vanishing along the year of 2006, demonstrating the high eddymonitoring capacity of the approach.

In this part, we applied the proposed on two different oceanic datasets interpolated to different spatial resolutions. In both simulations, the method shows high capacity of eddies' detection. In fact, the method works by identifying rotational segments as a first step and then uses a neighboring parameter $\epsilon$ to detect eddies. The first part relies on Lagrangian trajectories of particles. Thus, the finer the time step of advection is, the better the rotational segment extraction is. In this work, we used an $8 \mathrm{~h}$ time step, which is enough to reduce trajectories overshooting in oceanic data. On the other hand, the eddies' detection does not depend on the grid resolution but rather depends on the parameter $\epsilon$. In this work, we found an optimal choice of this parameter to be between three times and five times the size of the grid element.

\section{VALIDATION OF THE PROPOSED METHOD}

Here, we stretch the performance of the proposed method by comparing it with four recent methods from the Eulerian and Lagrangian frame. Through this comparison, we conduct a qualitative evaluation consisting of assessing the capacity of each method in identifying the genesis and splitting events of the eddies, the material coherency of its detected eddies, and a quantitative evaluation, based on the use of the same computational configuration, which consists in assessing the complexity and computational cost of each method in detecting all the eddies of 2006. We must note that some eddies may merge together or spin-down due to diffusive effects. However, such events are not identified by the proposed method, neither by the following ones. In this part, we use the same introductory conditions that are used to detect Lagrangian eddies from geostrophic data in the previous section. We also show the application of the Lagrangian methods to the simulated velocity field of the Navier-Stokes model 9 in the Appendix.

\section{A. Lagrangian methods}

\section{Lagrangian trajectory frequency}

The $\mathcal{L} \mathcal{T} \mathcal{F}$-based method $^{31}$ defines the Lagrangian vortex as a material line along which particles share similar frequency contents,

$$
\begin{gathered}
\mathcal{L T}_{\mathcal{T}} \mathcal{F}^{\xi}\left(\mathbf{x}\left(\cdot, t_{0}, \mathbf{x}_{0}\right)\right):=\int_{t_{0}}^{t_{n}} \mathbf{x}\left(t, \mathbf{x}_{0}\right) e^{-i t \xi} d t, \\
\xi=\left(\xi_{1}, \xi_{2}, \ldots \xi_{n}\right),
\end{gathered}
$$

where $\xi=\left(\xi_{1}, \xi_{2}, \ldots \xi_{n}\right)$ is a discrete set of $n$ frequencies of a given trajectory $\mathbf{x}\left(\cdot, t_{0}, \mathbf{x}_{0}\right)$. This method works by dumping the frequency content of Lagrangian trajectories $\mathbf{x}\left(t_{f}, t_{0}, \mathbf{x}_{0,1,2 . .}\right)$ into a scalar field $\bar{\xi}$, and then it detects vortices based on a convexity deficiency parameter $d$. The latter is defined as the ratio of the area difference between the vortex boundary and its convex hull to the area enclosed by the vortex boundary $d=\frac{A\left(\operatorname{conv}\left(E_{B}\left(t_{0}\right)-A\left(E_{B}\left(t_{0}\right)\right)\right)\right)}{A\left(E_{B}\left(t_{0}\right)\right)}$.

\section{Finite-time Lyapunov exponent}

The Lyapunov exponent is a classical measure to quantify the sensibility of a dynamical system to initial conditions,

$$
\lambda=\lim _{t \rightarrow \infty} \lim _{\delta(0) \rightarrow 0} \frac{1}{t} \log \frac{\delta(t)}{\delta 0}
$$

with $\delta(0)$ being the initial perturbation of a given trajectory and $\delta(t)$ the $t$ grown perturbation. To quantify the stretching of fluid parcels during a finite-time interval, the Lyapunov exponent is modified to a finite-time version, the finite-time Lyapunov exponent (FTLE),

$$
\lambda\left(t_{0}, \tau\right)=\frac{1}{\tau} \log \frac{\delta\left(t_{0}+\tau\right)}{\delta t_{0}}
$$

which measures the average exponential separation rate of trajectories with an initial distance $\delta\left(t_{0}\right)$ over a time period $\tau$ as $\delta(t)=\delta\left(t_{0}\right) e^{(\lambda t)}$. Positive FTLE indicates chaotic behavior, while in regular flows, the Lyapunov exponent is zero.

\section{Lagrangian-averaged vorticity deviation}

The LAVD-based vortex detection method developed in Ref. 45 is defined as the trajectory-averaged, normed deviation of the vorticity from its spatial mean,

$$
\operatorname{LAVD}_{t_{0}}^{t_{f}}\left(x_{0}\right)=\int_{t_{0}}^{t_{f}}\left|\omega\left(x\left(s ; x_{0}\right) s\right)-\bar{\omega}\right| d s,
$$

where $\bar{\omega}$ is the spatial mean of the vorticity $\omega$, which evolves according to Eq. (10). Similar to the $\mathcal{L} \mathcal{T} \mathcal{F}$, the $L A V D$-based method makes use of a convexity deficiency to identify the eddies boundaries as convex closed contours of high vorticity deviation.

For a single run, all the above methods are capable of identifying mesoscale eddies that are characterized by lifespans equal to the time interval of advection $\left[t_{0}, t_{f}\right]$, each based on the mapping of a certain Lagrangian quantity into a scalar field, and ensure their coherency during their time evolution without folding or stretching.

We start by applying these methods to the geostrophic velocity field; we set $d=10 \%, t_{0}=$ January 1,2006 , and vary $t_{f}$ each time to $t_{f}=t_{0}+(30,60,90,120)$. This allows us to compute, for each method, different scalar fields associated with different time intervals, as we show in Fig. 6. These methods' scalar fields present different Lagrangian quantities but highlight the same coherent structures in general. The $\mathcal{L} \mathcal{T} \mathcal{F}$ and $L A V D$ share the same procedure for eddies identification, whereas FTLE does not offer any procedure to their automatic identification, but only allows their visual identification. We show in Figs. 6(a) and 6(c) the different $\mathcal{L} \mathcal{T} \mathcal{F}$ and $L A V D$ scalar fields, along with the boundaries of their detected eddies (encircled in black and red colors). Boundaries labeled with the same number represent different cores (characterized by different lifetimes) of the same eddy. These methods do indeed identify the same eddies but extract different boundaries characterized by different diameters. Moreover, by varying the time interval of the flow maps, these methods seem to have different performances and $\mathcal{L} \mathcal{T} \mathcal{F}$ seems to identify more eddies while increasing the time interval of the flow map, contrary to $L A V D$, which show high eddies identification for shorter time intervals. The same remarks for the FTLE, and mesoscale eddies are well highlighted while increasing the time interval of the flow map. From a computational side, these methods seem to show good performance, for a single run over the year 2006, between $t_{0}=$ January 1 and $t_{f}=$ December 31 , 
a)
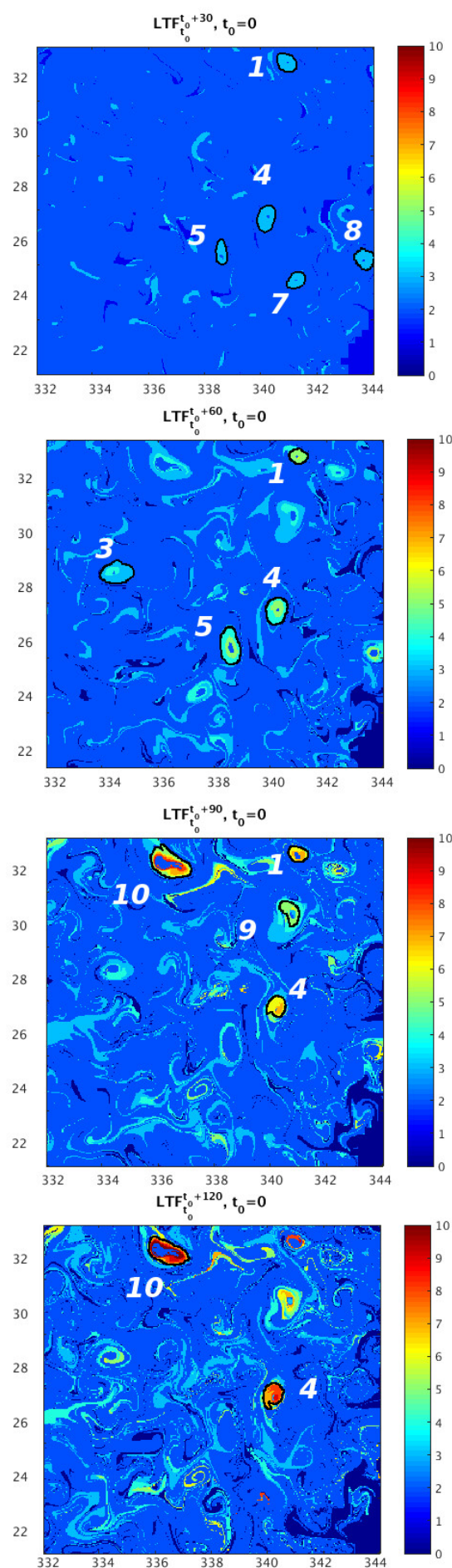

b)
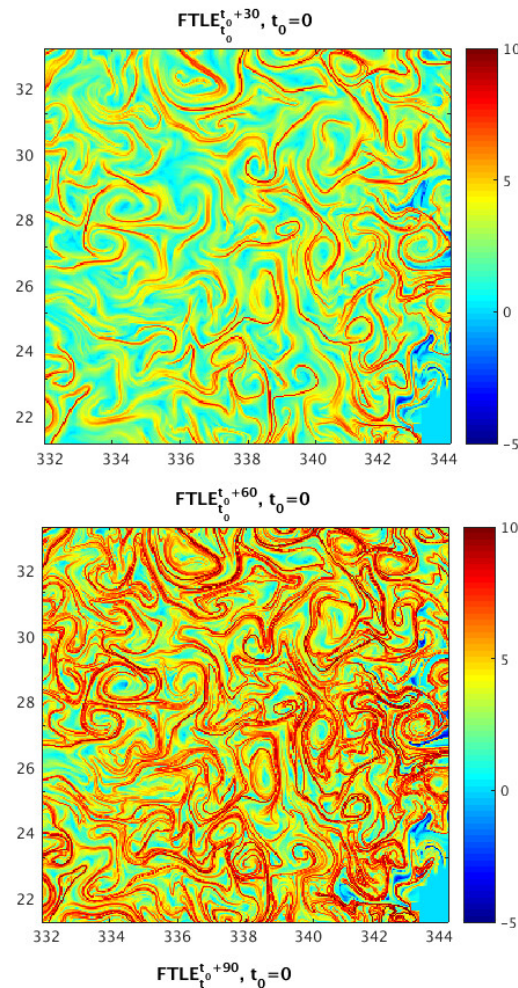

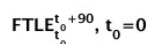
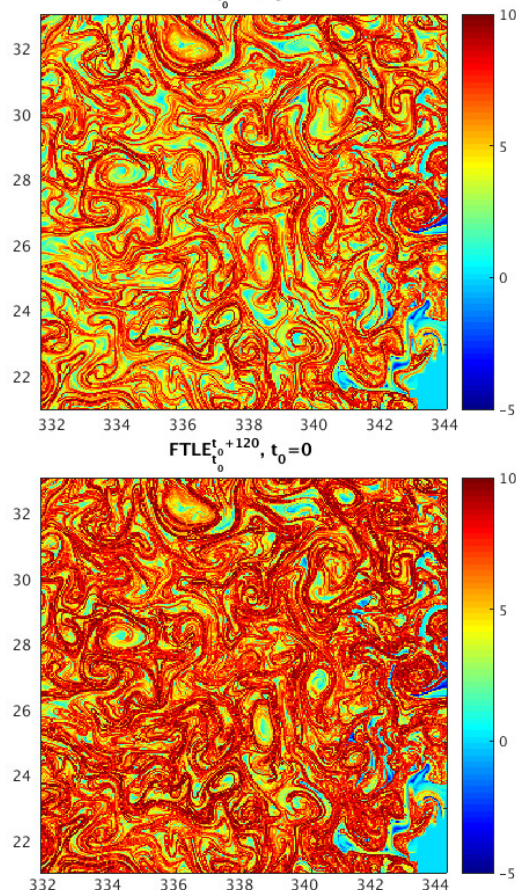

c)
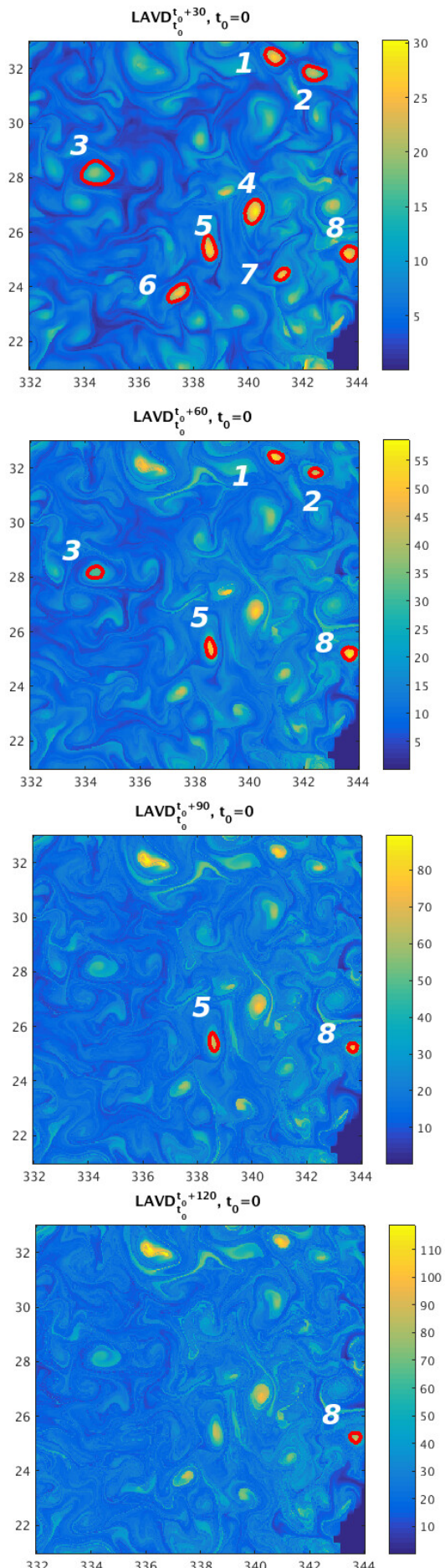

FIG. 6. $\mathcal{L} \mathcal{T} \mathcal{F}_{t_{0}}^{t_{f}}, F T L E_{t_{0}}^{t_{f}}$, and $L A V D_{t_{0}}^{t_{f}}$ fields computed for different time intervals: $\left[t_{0}, t_{0}+30\right.$ days], $\left[t_{0}, t_{0}+60\right.$ days], $\left[t_{0}, t_{0}+90\right.$ days], and $\left[t_{0}, t_{0}+120\right.$ days $]$. (a) $\mathcal{L} \mathcal{T} \mathcal{F}_{t_{0}}^{t_{f}}$ fields with detected mesoscale eddies being encircled with black color, and different cores of the same mesoscale eddy are labeled with the same number. (b) FTLE fields where eddies can be identified as homogeneous regions with separation rates close to zero. (d) LAVD fields with detected mesoscale eddies being encircled with red color. 
and the $L T F$ takes $51.14 \mathrm{~s}, 39.72 \mathrm{~s}$ for the $L A V D$ and $199.23 \mathrm{~s}$ for the FTLE. However, the main limitation of these methods is their reliance of the mapping of several Lagrangian quantities on a single scalar field, which makes it possible to detect only eddies that are coherent at $t_{0}$ and remain coherent until $t_{f}$, therefore missing several others that merged after $t_{0}$ and/or split before $t_{f}$. Therefore, by using these methods and in order to detect all the eddies that exist between $t_{0}$ $=$ January 1, 2006 and $t_{f}=$ December 31, 2006, including their genesis and splitting events, one has to follow the following procedure:
- Compute different $\mathcal{L} \mathcal{T} \mathcal{F}$ fields associated with different time intervals $\left[t_{i}, t_{j}\right]$, each time by varying $i$ from $0 . . n-1$ and $j$ from $1 . . n$, with $n$ being the number of discrete time steps, which results in a complexity of $\mathcal{O}\left(n^{2}\right)$.

- Once all eddies are identified, use an additional tool to track different cores of the same eddies to avoid their redundant.

Following just the first part of this procedure, without processing redundant of eddies, the $\mathcal{L} \mathcal{T} \mathcal{F}$ takes the overall value of $153 \mathrm{~min}$ and

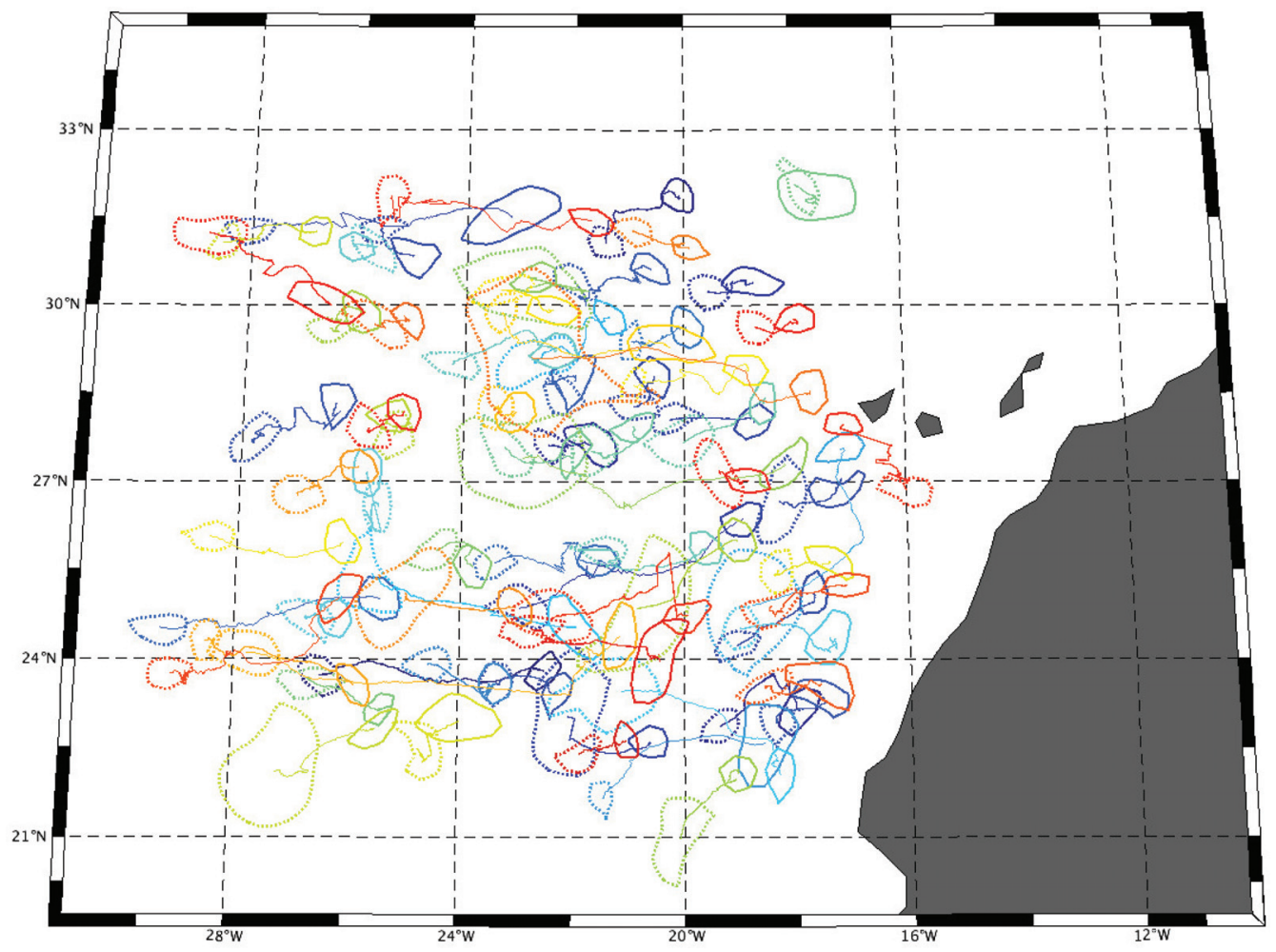

(a)

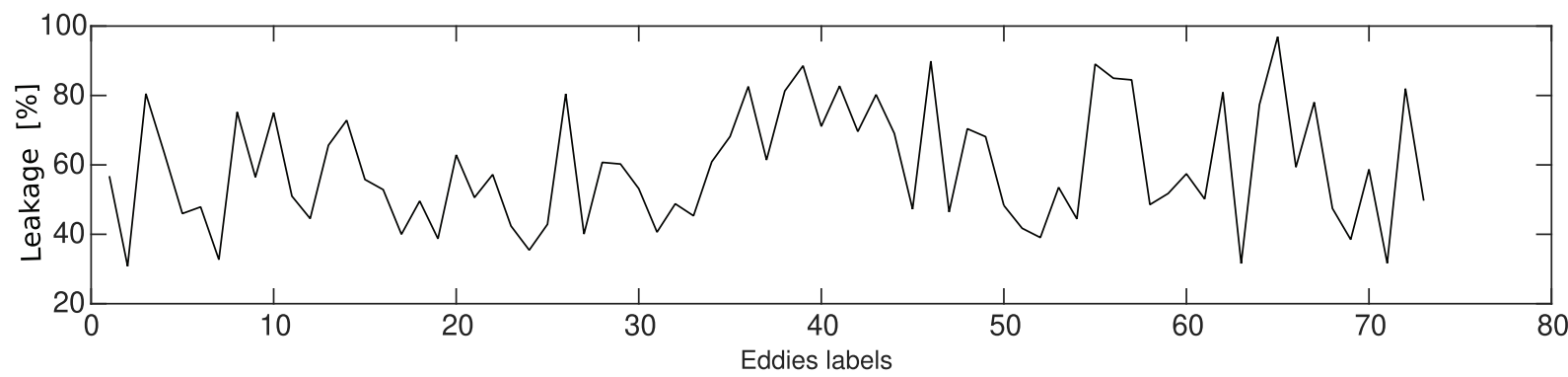

(b)

FIG. 7. (a) Initial and final (dashed line) positions of all the 74 Eulerian mesoscale eddies over the year 2006. Mesoscale eddies are presented in different colors following their genesis time. (b) Percentage of the leakage occurring through footprints of the detected eddies' boundaries and the Lagrangian advection of fluid parcels initialized at their time $t_{0}$ position. Multimedia view: http://dx.doi.org/10.1063/5.0038761.3 
TABLE I. Comparison of the performance of each method in identifying mesoscale eddies.

\begin{tabular}{|c|c|c|c|c|c|}
\hline & \multicolumn{5}{|c|}{ Methods } \\
\hline & $\begin{array}{l}\text { Proposed } \\
\text { method }\end{array}$ & $\begin{array}{l}\mathcal{L} \mathcal{T} \mathcal{F} \text {-based } \\
\text { method }\end{array}$ & $\begin{array}{l}\text { Okubo-Weiss } \\
\text { criterion }\end{array}$ & FTLE & LAVD \\
\hline Genesis and splitting events identification & Yes & No & Yes & No & No \\
\hline Ensures material coherency & Yes & Yes & No & Yes & Yes \\
\hline $\begin{array}{l}\text { Runtime between } 1 \text { January and } \\
31 \text { December } 2006\end{array}$ & $160.67 \mathrm{~s}$ & $51.14 \mathrm{~s}$ & $144.23 \mathrm{~s}$ & $199.23 \mathrm{~s}$ & $39.72 \mathrm{~s}$ \\
\hline Eddies detection from a single run & Yes & No & Yes & No & No \\
\hline Runtime to detect all eddies & $\mathcal{O}(1) 160.67 \mathrm{~s}$ & $\mathcal{O}\left(n^{2}\right) 153 \mathrm{~min}$ & $\mathcal{O}(1) 144.23 \mathrm{~s}$ & $\mathcal{O}\left(n^{2}\right) 599 \min$ & $\mathcal{O}\left(n^{2}\right) 119 \min$ \\
\hline Number of detected eddies & 64 & 49 & 74 & $\ldots$ & 52 \\
\hline
\end{tabular}

119 min for $L A V D$ and $599 \mathrm{~min}$ in the case of FTLE, which is very costly compared to $160.67 \mathrm{~s}$ achieved by the proposed method. Once the multiple scalar fields are computed for the $\mathcal{L} \mathcal{T} \mathcal{F}$ and $L A V D$ methods, we use an additional tool to avoid redundant of their detected eddies. A total of 49 eddies are detected by $\mathcal{L} \mathcal{T} \mathcal{F}$ and 52 eddies by the $L A V D$-based method. Although the numbers of their detected eddies are lower, however, they present the same one detected by the proposed method, but not necessarily the same core and lifetimes, mainly due to their reliance on the mapping of different Lagrangian quantities, whereas the proposed method analyzes the spatiotemporal dynamics of particle trajectories and defines eddies as group particles that share similar rotation, therefore presenting a robust basis to higheddy detection both in space and time.

\section{B. Okubo-Weiss criterion}

Here, we use the widely known Okubo-Weiss (OW) criterion ${ }^{26,27}$ to identify mesoscale eddies from the geostrophic data covering the year 2006 and use the method developed in Refs. 3 and 46 to track their motions. For a single run over the year 2006, this method detected 74 mesoscale eddies as we show in Fig. 7, which is an addition of 10 eddies compared to the proposed method. From a computational side, this method is very comparable to the proposed method and takes only $144.23 \mathrm{~s}$ to detect all the eddies of 2006. However, it uses instantaneous velocity fields to identify and track mesoscale eddies. Therefore, we must assess the material coherency of its detected eddies. We do this by passively advecting fluid parcels initialized at each eddy's time $t_{0}$ position, in forward time until the eddy's final time $t_{f}=t_{0}+$ lifetime. Then, we compute the portion of fluid parcels that ends up in the interior of each eddy's final Eulerian position. We evaluate a given eddy's leakage as the quantity difference between the fluid parcels initialized at the eddy's $t_{0}$ position and their advected images (at $t_{f}$ ) found in the eddy's $t_{f}$ position. We show in Fig. $7(\mathrm{~b})$ the percentage of the leakage occurring through the boundaries of the Eulerian eddies. In this way, they are found to lose roughly between $30 \%$ and $97 \%$ of their content while traveling westward. We display this leakage in movie M5 by showing the time evolution of these Eulerian footprint boundaries and the Lagrangian advection of their initial particles. Moreover, these Eulerian eddies form different cores, both in space and time, showing completely different eddies compared to those detected by the proposed method in the Lagrangian frame.
In this part, we evaluated the performance of the proposed method by comparing it with four methods from both Eulerian and Lagrangian frames. Through this comparison, we evaluated the computational cost of every method, its ability to identify the genesis and splitting event of the eddies, and the material coherency of their detected eddies. Table I summarizes this comparison. The proposed method has the advantage presented in the Eulerian method (Okubo-Weiss), which is the ability to identify the genesis and splitting events of the eddies. To our knowledge, existing Lagrangian eddy detection methods lack this property. The proposed method is Lagrangian, and therefore, it ensures material coherency of its detected eddies, which is not the case in the Eulerian method. Finally, it requires a low computational cost comparable to the Eulerian one.

\section{SUMMARY AND CONCLUSION}

Unlike the Eulerian methods, Lagrangian eddy detection methods are based on the analysis of a single scalar field that characterizes the fluid state that evolves along a "finite-time" interval. This is the mapping of several Lagrangian quantities (vorticity, trajectories' arc length, trajectories frequencies...) that evolve during an introductory interval of time $\left[t_{0}, t_{f}\right]$ into a scalar field, from which they identify eddies as distinguished regions characterized by different properties (higher vorticity, higher trajectories lengths, homogeneous frequencies...). These methods do indeed identify and track coherent eddies in a sharp manner. However, they only identify and detect eddies that (i) appear as coherent cores at the initial $t_{0}$ of the introductory interval $\left[t_{0}, t_{f}\right]$ and (ii) remain coherent all over the interval $\left[t_{0}, t_{f}\right]$. Therefore, they leave undetected several other eddies that form coherent cores after the initial time $t_{0}$ and other eddies with lifetimes shorter than the introductory interval $\left[t_{0}, t_{f}\right]$. Moreover, such a concept is not capable of identifying the genesis and splitting event of the detected eddies, therefore underestimating their lifetimes.

In this work, we proposed a robust Lagrangian eddy detection and tracking method, which is fully automatic and works by analyzing geometrical properties of the evolving velocity along Lagrangian trajectories and uses a grid density-based clustering algorithm to identify the genesis and splitting events of the eddies.

The proposed Lagrangian concept is the first among the existing Lagrangian methods to evaluate the state of the fluid at different subintervals from a single run, and it is capable of (i) identifying and extracting all mesoscale eddies from a single run (particles advection 
between $\left.\left[t_{0}, t_{f}\right]\right)$ regardless of their lifespans. Moreover, the method can be more selective by fixing the rotational segments' time length to a certain value $m_{t}$, thus detecting only mesoscale eddies with a specific lifetime, (ii) accurately following the dynamical evolution of the eddies and long-term water transport by (1) identifying the origin of the water forming the detected eddies, (2) extracting the eddies cores at their genesis time, (3) tracking their coherent cores until their splitting event, and (4) tracking and quantifying the impact of their trapped water after they lose their coherency. This is crucial for quantifying coherent transport of water properties such as heat, salt, and biogeochemical species.

This study focuses on providing a novel eddy detection method with high-monitoring capacity. The method was demonstrated visually by applying it on an oceanic model velocity field, and its performance was tested by detecting and tracking accurately different mesoscale eddies off the Canary system from the geostrophic velocity field derived from satellite altimetry. The method was also compared with four recent methods from the Eulerian and Lagrangian frame to validate its high eddy monitoring capacity. In the future, the method presented here will be applied on a global scale, with an aim toward characterizing different properties of mesoscale eddies and quantifying their coherent transport.

\section{APPENDIX: THE PERFORMANCE OF THE LAGRANGIAN METHODS OVER THE DIRECT FLUID SIMULATION}

Here, we apply the FTLE, LAVD, and $\mathcal{L} \mathcal{T} \mathcal{F}$ methods on the simulated velocity field of the Navier-Stokes model 9 . We compute for each method three scalar fields associated with the lifespan of each simulated vortex: $\left[t_{0}=0, t_{f}=370\right],\left[t_{0}=30, t_{f}=160\right]$, and $\left[t_{0}=66, t_{f}=128\right]$. We show in Figs. $8(\mathrm{a})-8(\mathrm{c})$ the different scalar
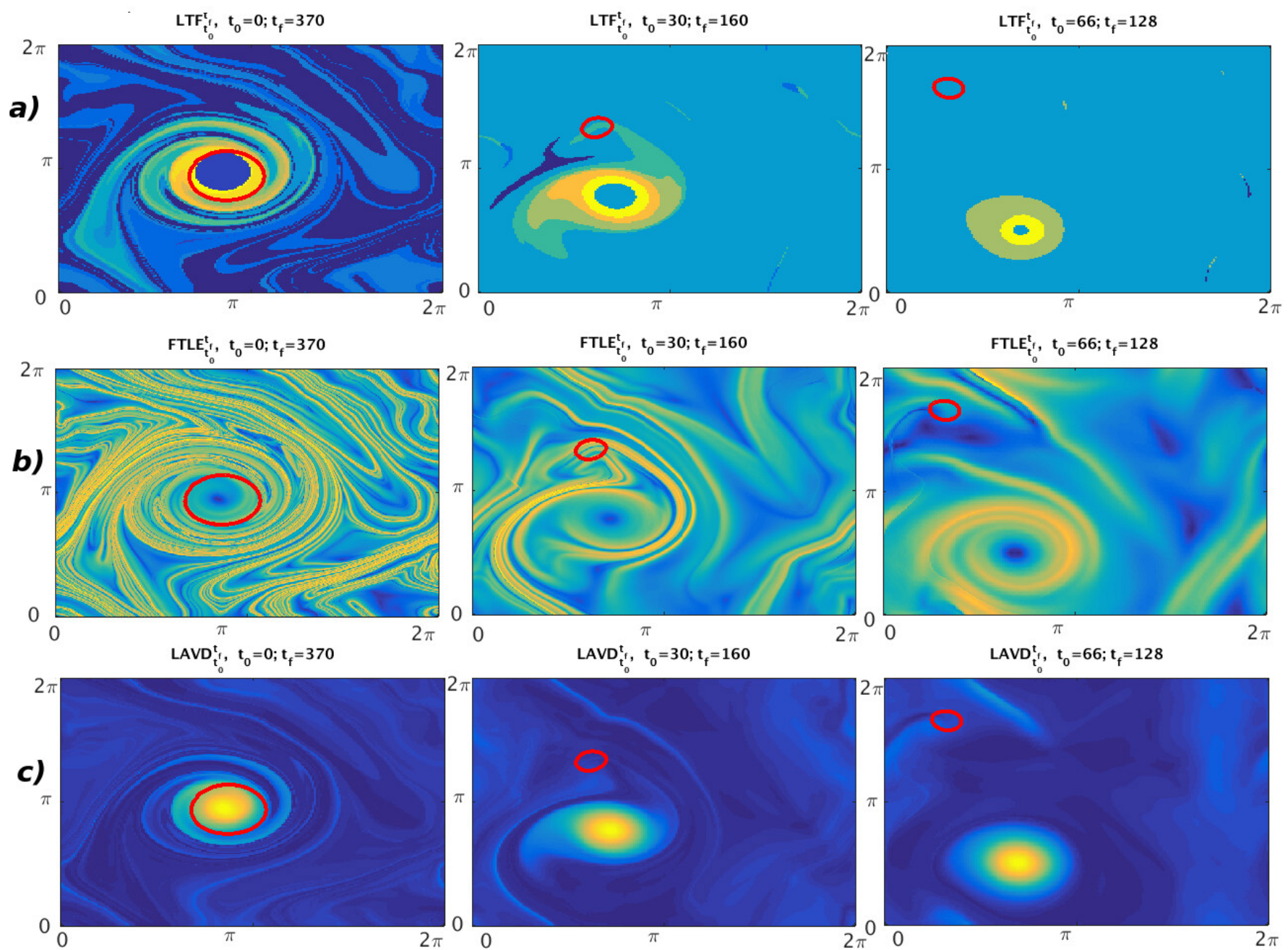

FIG. 8. (a) $\mathcal{L} \mathcal{T} \mathcal{F}_{t_{f}}^{t_{f}}$, (b) $F T L E_{t}^{t_{t}}$, and (c) $L A V D_{t}^{t_{t}}$ fields computed for the direct simulation 9 at different time intervals. In red lines, the boundaries of the three detected vortices identified by the proposed method at $t=0, t=30$, and $t=66$. 
fields of each method in the background and the boundaries of each vortex as detected by the proposed method. We see that all methods are capable of identifying the larger vortex. However, none of them is capable of identifying the other two.

These methods identify vortices by the mapping of several Lagrangian quantities of the flow map $\Phi_{t_{0}}^{t_{f}}$ into a scalar field. The $\mathcal{L} \mathcal{T} \mathcal{F}$ uses the frequency contents of the Lagrangian trajectories, the FTLE uses the separation rate of initial nearby trajectories, and the LAVD method uses the Lagrangian vorticity deviation to identify eddies. By this example, we showed that the mapping of such quantities might not be enough to identify and detect coherent vortices. On the other hand, the proposed method does identify all these vortices from a single run, showing high vortex monitoring capacity.

\section{DATA AVAILABILITY}

The surface geostrophic velocity data that support the findings of this study are provided daily on $1 / 4^{\circ}$ resolution in the Copernicus Marine Environment Monitoring Service at https://marine.copernicus.eu, https://resources.marine.copernicus.eu/documents/PUM/CMEMSSL-PUM-008-032-062.pdf.

\section{REFERENCES}

${ }^{1}$ R. Ferrari and C. Wunsch, "Ocean circulation kinetic energy: Reservoirs, sources, and sinks," Annu. Rev. Fluid Mech. 41, 253 (2009).

${ }^{2}$ S. Schemm, S. Rüdisühli, and M. Sprenger, "The life cycle of upper-level troughs and ridges: A novel detection method, climatologies and Lagrangian characteristics," Weather Clim. Dyn. 1, 459-479 (2020).

${ }^{3}$ R. Escudier, L. Renault, A. Pascual, P. Brasseur, D. Chelton, and J. Beuvier, "Eddy properties in the Western Mediterranean sea from satellite altimetry and a numerical simulation," J. Geophys. Res.: Oceans 121, 3990-4006, https:// doi.org/10.1002/2015JC011371 (2016).

${ }^{4}$ G. Dencausse, M. Arhan, and S. Speich, "Routes of Agulhas rings in the Southeastern cape basin,” Deep Sea Res. Part I 57, 1406-1421 (2010).

${ }^{5}$ V. Rossi, C. López, E. Hernández-García, J. Sudre, V. Garçon, and Y. Morel, "Surface mixing and biological activity in the four Eastern Boundary Upwelling Systems," Nonlinear Processes Geophys. 16(4), 557-568 (2009).

${ }^{6}$ F. d'Ovidio, V. Fernández, E. Hernández-García, and C. López, "Mixing structures in the Mediterranean sea from finite-size Lyapunov exponents," Geophys. Res. Lett. 31, L17203, https://doi.org/10.1029/2004GL020328 (2004).

${ }^{7}$ A. El Aouni, K. Daoudi, H. Yahia, K. Minaoui, and A. Benazzouz, "Surface mixing and biological activity in the North-West African Upwelling," Chaos 29, 011104 (2019).

${ }^{8}$ D. J. McGillicuddy, Jr., A. R. Robinson, D. A. Siegel, H. W. Jannasch et al., "Influence of mesoscale eddies on new production in the Sargasso Sea," Nature 394, 263 (1998).

${ }^{9}$ A. Oschlies and G. Veronique, "Eddy-induced enhancement of primary production in a model of the North Atlantic Ocean," Nature 394, 266 (1998).

${ }^{10}$ E. T. Kai, V. Rossi, J. Sudre, H. Weimerskirch, C. Lopez, E. Hernandez-Garcia, F. Marsac, and V. Garçon, "Top marine predators track Lagrangian coherent structures," Proc. Natl. Acad. Sci. 106, 8245-8250 (2009).

${ }^{11}$ C. Cotté, F. d'Ovidio, A. Chaigneau, M. Lévy, I. Taupier-Letage, B. Mate, and C. Guinet, "Scale-dependent interactions of Mediterranean whales with marine dynamics," Limnol. Oceanogr. 56, 219-232 (2011).

${ }^{12}$ M. Hassan, L. C. Hendrickson, A. Arkhipkin, G. J. Pierce, A. Gangopadhyay, H. Kidokoro, U. Markaida, C. Nigmatullin, W. H. Sauer, P. Jereb et al., "Ecological-fishery forecasting of squid stock dynamics under climate variability and change: Review, challenges, and recommendations," Rev. Fisheries Sci. Aquaculture 1-36 (2020).

${ }^{13}$ I. Frenger, N. Gruber, R. Knutti, and M. Münnich, "Imprint of Southern ocean eddies on winds, clouds and rainfall,” Nat. Geosci. 6, 608-612 (2013).
${ }^{14} \mathrm{~J}$. C. McWilliams, “The vortices of two-dimensional turbulence," J. Fluid Mech. 219, 361-385 (1990).

${ }^{15}$ A. M. Doglioli, B. Blanke, S. Speich, and G. Lapeyre, "Tracking coherent structures in a regional ocean model with wavelet analysis: Application to cape basin eddies,” J. Geophys. Res. 112, C05043, https://doi.org/10.1029/2006JC003952 (2007).

${ }^{16}$ Z. Kizner, B. Shteinbuch-Fridman, V. Makarov, and M. Rabinovich, "Cycloidal meandering of a mesoscale anticyclonic eddy,” Phys. Fluids 29, 086601 (2017).

${ }^{17}$ Z. Wang, K. Luo, D. Li, J. Tan, and J. Fan, "Investigations of data-driven closure for subgrid-scale stress in large-eddy simulation," Phys. Fluids 30, 125101 (2018).

${ }^{18}$ L. Zhang, Q. Deng, R. Machiraju, A. Rangarajan, D. Thompson, D. K. Walters, and H.-W. Shen, "Boosting techniques for physics-based vortex detection," in Computer Graphics Forum (Wiley Online Library, 2014), Vol. 33, pp. 282-293.

${ }^{19}$ X. Dong, Y. Gao, and C. Liu, "New normalized rortex/vortex identification method," Phys. Fluids 31, 011701 (2019).

${ }^{20}$ CQun Liu, Y. Wang, Y. Yang, and ZWei Duan, "New omega vortex identification method,” Sci. China Phys., Mech. Astron. 59, 684711 (2016).

${ }^{21}$ J. Liu and C. Liu, "Modified normalized rortex/vortex identification method," Phys. Fluids 31, 061704 (2019).

${ }^{22}$ C. Liu, Y. Gao, S. Tian, and X. Dong, "Rortex-A new vortex vector definition and vorticity tensor and vector decompositions," Phys. Fluids 30, 035103 (2018).

${ }^{23}$ Y. Gao and C. Liu, "Rortex based velocity gradient tensor decomposition," Phys. Fluids 31, 011704 (2019).

${ }^{24}$ A. E. Perry and M. S. Chong, "A description of eddying motions and flow patterns using critical-point concepts,” Annu. Rev. Fluid Mech. 19, 125-155 (1987).

${ }^{25}$ J. Zhou, R. J. Adrian, S. Balachandar, and T. M. Kendall, "Mechanisms for generating coherent packets of hairpin vortices in channel flow," J. Fluid Mech. 387, 353-396 (1999).

${ }^{26} \mathrm{~A}$. Okubo, "Horizontal dispersion of floatable particles in the vicinity of velocity singularities such as convergences," in Deep Sea Research and Oceanographic Abstracts (Elsevier, 1970), Vol. 17, pp. 445-454.

${ }^{27}$ J. Weiss, "The dynamics of enstrophy transfer in two-dimensional hydrodynamics," Physica D 48, 273-294 (1991).

${ }^{28}$ J. H. Elsas Luca Moriconi, "Vortex identification from local properties of the vorticity field," Phys. Fluids 29, 015101 (2017).

${ }^{29}$ I. Ari Sadarjoen and F. H. Post, "Detection, quantification, and tracking of vortices using streamline geometry," Comput. Graph. 24, 333-341 (2000).

${ }^{30}$ A. Chaigneau, A. Gizolme, and C. Grados, "Mesoscale eddies off Peru in altimeter records: Identification algorithms and eddy spatio-temporal patterns," Prog. Oceanogr. 79, 106-119 (2008).

${ }^{31}$ A. El Aouni, H. Yahia, K. Daoudi, and K. Minaoui, "A Fourier approach to Lagrangian vortex detection,” Chaos 29, 093106 (2019).

${ }^{32}$ A. El Aouni, K. Daoudi, H. Yahia, S. K. Maji, and K. Minaoui, "Defining Lagrangian coherent vortices from their trajectories," Phys. Fluids 32, 016602 (2020).

${ }^{33}$ S. Katsanoulis, M. Farazmand, M. Serra, and G. Haller, "Vortex boundaries as barriers to diffusive vorticity transport in two-dimensional flows," Phys. Rev. Fluids 5, 024701 (2020).

${ }^{34}$ E. L. Rempel, A. C.-L. Chian, F. J. Beron-Vera, S. Szanyi, and G. Haller, "Objective vortex detection in an astrophysical dynamo," Mon. Not. R. Astron. Soc. 466, L108-L112 (2017).

${ }^{35}$ N. O. Aksamit, T. Sapsis, and G. Haller, "Machine-learning mesoscale and submesoscale surface dynamics from Lagrangian ocean drifter trajectories," J. Phys. Oceanogr. 50, 1179-1196 (2020).

${ }^{36}$ A. Hadjighasem, D. Karrasch, H. Teramoto, and G. Haller, "Spectral-clustering approach to Lagrangian vortex detection," Phys. Rev. E 93, 063107 (2016).

${ }^{37}$ G. Froyland, C. P. Rock, and K. Sakellariou, "Sparse eigenbasis approximation: Multiple feature extraction across spatiotemporal scales with application to coherent set identification," Commun. Nonlinear Sci. Numer. Simul. 77, 81-107 (2019).

${ }^{38}$ G. Froyland, "An analytic framework for identifying Finite-Time coherent sets in time-dependent dynamical systems," Physica D 250, 1-19 (2013). 
${ }^{39}$ G. Froyland, C. Horenkamp, V. Rossi, N. Santitissadeekorn, and A. S. Gupta, "Three-dimensional characterization and tracking of an Agulhas ring," Ocean Modell. 52-53, 69-75 (2012).

${ }^{40}$ G. Froyland, P. Koltai, and M. Stahn, "Computation and optimal perturbation of Finite-Time coherent sets for aperiodic flows without trajectory integration," SIAM J. Appl. Dyn. Syst. 19, 1659-1700 (2020).

${ }^{41}$ H.-P. Kriegel, P. Kröger, J. Sander, and A. Zimek, "Density-based clustering," Wiley Interdiscip. Rev.: Data Min. Knowl. Discovery 1, 231-240 (2011).

${ }^{42}$ W. F. Spotz and G. F. Carey, "High-order compact scheme for the steady stream-function vorticity equations," Int. J. Numer. Methods Eng. 38, 3497-3512 (1995)
${ }^{43}$ A. El Aouni, V. Garçon, J. Sudre, H. Yahia, K. Daoudi, and K. Minaoui, "Physical and biological satellite observations of the northwest african upwelling: Spatial extent and dynamics," IEEE Trans. Geosci. Remote Sens. 58, 1409-1421 (2020).

${ }^{44} \mathrm{P}$. Jan van Leeuwen, "The propagation mechanism of a vortex on the $\beta$ plane," J. Phys. Oceanogr. 37, 2316-2330 (2007).

${ }^{45}$ G. Haller, A. Hadjighasem, M. Farazmand, and F. Huhn, "Defining coherent vortices objectively from the vorticity," J. Fluid Mech. 795, 136-173 (2016).

${ }^{46}$ D. B. Chelton, M. G. Schlax, and R. M. Samelson, "Global observations of nonlinear mesoscale eddies,” Prog. Oceanogr. 91, 167-216 (2011). 